\title{
ESTIMAÇÃO DE MAGNITUDE DO ESTRESSE, PELOS ALUNOS DE GRADUAÇÃO, QUANTO AO CUIDADO DE ENFERMAGEM
}

\author{
Renata Alessandra Evangelista ${ }^{\top}$ \\ Priscilla Hortense ${ }^{2}$ \\ Fátima Aparecida Emm Faleiros Sousa ${ }^{3}$
}

Evangelista RA, Hortense P, Sousa FAEF. Estimação de magnitude do estresse, pelos alunos de graduação, quanto ao cuidado de enfermagem. Rev Latino-am Enfermagem 2004 novembro-dezembro; 12(6):913-7.

Estresse é definido como uma resposta fisiológica, psicológica e comportamental de um indivíduo que procura adaptarse e ajustar-se às pressões internas e externas. O objetivo foi verificar o estresse do aluno de enfermagem frente aos cuidados realizados. Utilizamos métodos psicofísicos diretos, escala de razão e emparelhamento intermodal. A amostra constou de 15 discentes, matriculados no $3^{\circ}$ semestre de enfermagem de uma instituição de ensino. Foi realizada a validação psicofísica da escala de razão dos cuidados de enfermagem através do método de emparelhamento intermodal. Os dados foram coletados usando-se questionário com 15 cuidados de enfermagem, em que a tarefa do aluno consistia em estimar um número a cada cuidado que fosse proporcional à quantidade de estresse que essa proporciona ao ser executada. Obteve-se, como resultado, que a sondagem vesical de demora é considerada o cuidado mais estressante, e que a verificação de temperatura foi considerado o cuidado menos estressante. É necessário a continuidade dos estudos, oferecendo outras comparações e com outras amostras de população.

DESCRITORES: estresse; cuidados de enfermagem; estudantes de enfermagem

\section{MAGNITUDE ESTIMATION OF STRESS IN VIEW OF NURSING CARE}

Stress is defined as the physiological, psychological and behavioral response of an individual attempting to adapt to internal and external pressure. This study aimed at observing how nursing students perceive stress in view of the care delivered in hospitals. Direct psychophysical methods, ratio scale and cross-modality matching were used. The sample consisted of 15 students registered in the third term of a nursing program. The ratio scale for nursing care was subject to psychophysical validation through the cross-modality matching method. Data were collected by means of a questionnaire containing 15 types of nursing care delivered at hospitals, in which the task consisted in estimating a number for each type, which would be proportional to the amount of stress caused during its performance. Results showed that permanent vesical probing is considered the most stressful type of care, followed by relief vesical probing, and that temperature taking was regarded as the least stressful technique. There is a need for further study, through other comparisons and with other population samples.

DESCRIPTORS: stress; nursing care; students, nursing

\section{ESTIMACIÓN DE MAGNITUD DE ESTRÉS FRENTE A LOS CUIDADOS DE ENFERMERÍA}

Estrés es definido como una respuesta fisiológica, psicológica y comportamental de un individuo que procura adaptarse y ajustarse a las presiones internas y externas. El objetivo de este estudio fue verificar la percepción del estrés del alumno de enfermería frente al cuidado realizado en el hospital. Fueron utilizados métodos psicofísicos directos, escala de razón y método de emparejamiento intermodal. La muestra fue constituida por 15 alumnos, matriculados en el $3^{\circ}$ semestre de enfermería de una institución de enseñanza. Fue efectuada validación psicofísica de la escala de razón del cuidado de enfermería a través del método de emparejamiento intermodal. Los datos fueron recopilados mediante un cuestionario con 15 cuidados de enfermería realizados en el hospital, en que la tarea representaba estimar un número para cada cuidado que fuese proporcional a la cantidad de estrés proporcionada en su ejecución. Se obtuvo como resultado que el sondaje vesical permanente es considerado el cuidado más estresante, seguido del sondaje vesical temporal, y que la verificación de la temperatura fue considerada el cuidado menos estresante. Es necesaria la continuidad de los estudios, ofreciendo otras comparaciones y con otras muestras de población.

DESCRIPTORES: estrés; atención de enfermería; estudiantes de enfermería

\footnotetext{
${ }^{1}$ Mestre, Docente da Universidade de Araraquara; ${ }^{2}$ Mestre; ${ }^{3}$ Professor Associado da Escola de Enfermagem de Ribeirão Preto, da Universidade de São Paulo, Centro Colaborador da OMS para o desenvolvimento da pesquisa em enfermagem, e-mail: faleiros@eerp.usp.br
} 
INTRODUÇÃO

Odesenvolvimento da habilidade psicomotora no estudante de enfermagem se faz através da aprendizagem de cuidados que são, na verdade, várias atividades psicomotoras utilizadas para se prestar assistência de enfermagem ${ }^{(1)}$.

Os cuidados são aprendidos a partir e durante o Curso de Graduação em Enfermagem. Grande parte das técnicas, ou pelo menos as básicas, são aprendidas na primeira disciplina desse curso, porque é nela que se desenvolve o primeiro contato do aluno com o processo de assistir o paciente. Além da prática, outro aspecto importante que influencia a aprendizagem das habilidades é o estresse.

O primeiro pesquisador em estresse, Hans Selye, que é considerado o pai dessa linha de pesquisa, é médico endocrinologista. A palavra estresse designa o conjunto dos efeitos não específicos de fatores que podem agir sobre o corpo, é o estado manifestado por uma síndrome específica constituída por todas as alterações não específicas produzidas num sistema biológico, em resumo, é uma reação inespecífica do corpo a qualquer demanda ${ }^{(2-}$ ${ }^{3)}$. Estressor é todo o agente que produz estresse; conceito que posteriormente foi melhor especificado por outros autores, no sentido de defini-lo como qualquer agente físico, químico, biológico ou psicológico, tanto de natureza intrínseca quanto extrínseca do sujeito ${ }^{(4)}$.

A enfermagem é reconhecida mundialmente como uma profissão estressante. É alvo de diferentes pesquisas por diversos focos de atenção e por outros profissionais. Alguns autores detectaram a existência de várias características evidenciáveis de estresse em discentes de enfermagem, algumas em maior, outras em menor intensidade, dentre elas a angústia, taquicardia, distúrbios gastrointestinais, entre outras ${ }^{(5-6)}$. Um outro autor estudou uma população de enfermeiros americanos e chegou à descrição do burnout como uma síndrome que ocorre com o profissional e sua falta de adaptação às condições e dinâmica de trabalho ${ }^{(7)}$.

Compreendendo a importância de se mensurar o nível de stress e/ou reajustamento social, além de sua intensidade ${ }^{(8)}$, alguns autores ${ }^{(9)}$ elaboraram uma escala contendo 43 itens de eventos de vida que requeriam diferentes graus de reajustamentos. Alguns desses itens foram: casamento, morte da esposa, gravidez, mudança de residência, dificuldades sexuais, divórcio, problemas no emprego, férias, entre outros. Essa escala foi denominada de escala de magnitudes de reajustamentos sociais. A tarefa dos participantes constitui em dar uma estimativa de magnitude para cada um dos itens dessa escala que refletisse o grau relativo de reajustamento social (estresse) necessário para se acomodar a esse evento, independente da desejabilidade do mesmo. O evento de vida "casamento" foi tomado como estímulo padrão e a ele foi designado o valor de 500 (módulo). Os outros eventos deveriam ser estimados proporcionalmente ao casamento tomado como padrão. Os resultados foram extremamente consistentes e invariáveis. De fato, dezesseis coeficientes de correlações produto-momento, envolvendo combinações entre as variáveis idade, sexo, estado civil, educação, classe social, religião, raça, gerações, variavam de 0,82 a 0,98, com coeficiente médio de $0,95^{(10)}$.

Além disso, a escala de estimação de magnitudes de reajustamentos sociais tem sido amplamente utilizada em diferentes contextos, como o hospitalar e com diferentes faixas etárias, como na velhice.

Portanto, não há estudos no Brasil que têm investigado a percepção do estresse frente aos cuidados de enfermagem, realizados por esse profissional, utilizando-se de procedimentos usados em psicofísica sensorial. No entanto, ressalta-se a importância de um estudo experimental com mensuração em nível de razão, uma vez que a enfermagem, enquanto ciência, necessita utilizar instrumentos de observação, experimentação e mensuração mais precisas e com dados replicáveis.

\section{OBJETIVOS}

- Escalonar os descritores atribuídos aos cuidados realizados pelo profissional de enfermagem.

- Verificar se as ordenações dos graus de estresse, derivada das duas modalidades de medida, são similares entre si.

- Verificar se a variabilidade das estimativas de magnitude e daquelas de comprimento de linhas é uma função linear das médias geométricas dessas estimativas, ou seja, seguem a Lei de Ekman, tal como usualmente verificada para contínuos métricos.

- Validar a escala de razão derivada para o contínuo não- 
métrico de atribuição de estresse frente ao cuidado de enfermagem, através do método de emparelhamento intermodal.

- Verificar a estabilidade e/ou equivalência dessas escalas de razão produzidas através de duas modalidades de respostas diferentes, quais sejam numérica sem limites (estimativas de magnitudes) e visual (comprimento de linhas).

\section{MÉTODO}

Participantes

Participaram 15 alunos do curso de graduação em Enfermagem de uma universidade da cidade de Ribeirão Preto que estavam cursando a primeira disciplina do ciclo profissionalizante, com idades entre 18 e 23 anos. Todos os participantes eram ingênuos quanto aos propósitos do experimento, ou seja, os sujeitos não tinham experiência prévia com experimentos dessa natureza.

Material

Foram montados dois blocos de papel contendo, na primeira página, instruções específicas para cada tipo de método psicofísico utilizado e, nas páginas seguintes, uma lista contendo 15 cuidados de enfermagem.

Procedimento

Os métodos psicofísicos utilizados foram estimação de magnitudes e emparelhamento intermodal envolvendo o contínuo de resposta de comprimento de linhas. No primeiro método, a tarefa dos participantes foi dar um número a cada cuidado que fosse proporcional à quantidade de estresse que aquele cuidado proporciona ao ser executado, em relação ao primeiro cuidado apresentado. Dessa forma, se o participante julgasse que um dado cuidado acarretasse duas vezes mais estresse do que aquele cuidado, deveria assinalar um número duas vezes maior. Se julgar que um dado cuidado possuísse metade do estresse do que o outro, ele deveria assinalar um número que fosse metade do atribuído àquele cuidado. Cada participante estabeleceu estimativas para os 15 cuidados de enfermagem. A técnica SOROTERAPIA foi tomada como estímulo padrão e recebeu o módulo de 100.

Com o método de emparelhamento intermodal, envolvendo o contínuo de resposta de comprimento de linhas, a tarefa dos participantes consistiu em emparelhar um comprimento de linha a cada descritor que fosse proporcional à quantidade de atribuição de estresse frente aos cuidados de enfermagem. Dessa forma, se o participante julgasse que um dado cuidado leva duas vezes mais estresse do que aquele cuidado, deveria emparelhar a ele um comprimento de linha duas vezes maior. Se julgasse que um dado cuidado possui metade do estresse do que aquele cuidado, deveria assinalar a ele um comprimento de linha que fosse metade do atribuído àquele cuidado. Os diferentes cuidados de enfermagem foram apresentados em uma série de 15 , os quais foram dispostos em uma página, numa ordem totalmente aleatória para todos os participantes. Cada participante estabeleceu 15 estimativas, sendo 1 para cada cuidado de enfermagem. Estímulo padrão e módulo foram previamente designados, sendo o estímulo padrão SOROTERAPIA e o módulo 100.

Nas instruções dadas para os participantes, independente do método psicofísico utilizado, foi solicitado que os julgamentos fossem realizados em termos do conhecimento teórico e/ou prático adquirido a respeito dos cuidados de enfermagem em questão. O experimento foi realizado nas salas de aula ou nos laboratórios, de acordo com a facilidade dos participantes e esses eram orientados a fazer os julgamentos individualmente.

Os mesmos 15 participantes fizeram as duas tarefas, sendo que metade julgou primeiro pelo método de estimação de magnitudes e depois pelo método de emparelhamento intermodal, envolvendo contínuo de resposta de comprimento de linha.

\section{RESULTADOS E DISCUSSÃO}

As estimativas médias para os diferentes cuidados de enfermagem, realizados pelos participantes e as respectivas ordenações de posições, estão apresentadas na Tabela 1 para os psicofísicos de estimação de magnitudes (EM) e de emparelhamento de comprimentos de linhas (EMCL). 
Tabela 1 - Média geométrica das estimativas de magnitudes (EM), média geométrica dos emparelhamentos de comprimentos de linhas (EMCL) e respectivas ordenações de posições (OP) de atribuição julgadas de cada cuidado de enfermagem

\begin{tabular}{|c|c|c|c|c|}
\hline Técnicas de enfermagem & EM & OP & EMCL & OP \\
\hline $\begin{array}{l}\text { Sondagem vesical de } \\
\text { demora }\end{array}$ & 141,55 & $1^{\circ}=$ & 108,55 & $1^{\circ}$ \\
\hline Lavagem intestinal & 129,48 & $4^{0}$ & 87,77 & $5^{\circ}$ \\
\hline Sondagem nasogástrica & 131,88 & $3^{\circ}$ & 88,04 & $4^{\circ}$ \\
\hline $\begin{array}{l}\text { Sondagem vesical de } \\
\text { alívio }\end{array}$ & 135,42 & $2^{0}$ & 83,50 & $7^{\circ}$ \\
\hline $\begin{array}{l}\text { Medicamentos via } \\
\text { endovenosa }\end{array}$ & 128,28 & $6^{0}$ & 84,05 & $6^{\circ}$ \\
\hline Soroterapias & 100,00 & $8^{\circ}$ & 100,00 & $2^{0}$ \\
\hline Fleet enemas & 129,48 & $5^{\circ}$ & 76,95 & $8^{\circ}$ \\
\hline Colheita de sangue & 118,22 & $7^{0}$ & 90,25 & $3^{\circ}$ \\
\hline $\begin{array}{l}\text { Medicamentos } \\
\text { intramuscular }\end{array}$ & 62,39 & $11^{\circ}$ & 75,15 & 9o \\
\hline Banho no leito & 64,09 & $10^{\circ}$ & 55,69 & $11^{\circ}$ \\
\hline Alimentação por SNG & 83,72 & $9^{\circ}$ & 74,11 & $10^{\circ}$ \\
\hline $\begin{array}{l}\text { Colocação de luvas } \\
\text { estéreis }\end{array}$ & 24,17 & $14^{\circ}$ & 22,18 & $14^{\circ}$ \\
\hline Oxigenoterapia & 39,57 & $12^{\circ}$ & 28,21 & $13^{\circ}$ \\
\hline Arrumação no leito & 36,14 & $13^{\circ}$ & 31,51 & $12^{\circ}$ \\
\hline $\begin{array}{l}\text { Verificação de } \\
\text { temperatura } \\
\end{array}$ & 9,91 & $15^{\circ}$ & 8,29 & $15^{\circ}$ \\
\hline
\end{tabular}

Pode-se observar na Tabela 1 que, independente do método psicofísico utilizado, os cuidados sondagem vesical de demora, sondagem nasogástrica e lavagem intestinal foram considerados aqueles de maior atribuição de estresse, enquanto que os cuidados verificação de temperatura, colocação de luvas estéreis e arrumação do leito os de menor atribuição. Por exemplo, o cuidado sondagem vesical de demora ocupa a 1 a posição, o cuidado de administração de medicamentos endovenosa ocupa a $6^{\underline{a}}$ e 0 cuidado colocação de luvas estéreis ocupa a $14^{a}$ posição nos dois contínuos de respostas.

Outro resultado interessante, considerando as duas amostras juntas, é a relação entre os desvios padrão da média geométrica e as médias geométricas dos emparelhamentos de comprimentos de linhas (ver Figura 1). Observa-se que a relação é linear, de modo que quanto maior a estimativa de magnitude (ou emparelhamento de comprimento de linha) tanto maior é o desvio padrão, ou seja, a variabilidade das estimativas é uma função linear da magnitude das estimativas (ou emparelhamentos), mostrando assim o princípio geral conhecido como lei de Ekman.

Do ponto de vista psicofísico, portanto, essa grande similaridade comprova que a escala é válida, ou seja, os expoentes seguem a propriedade de transitividade.

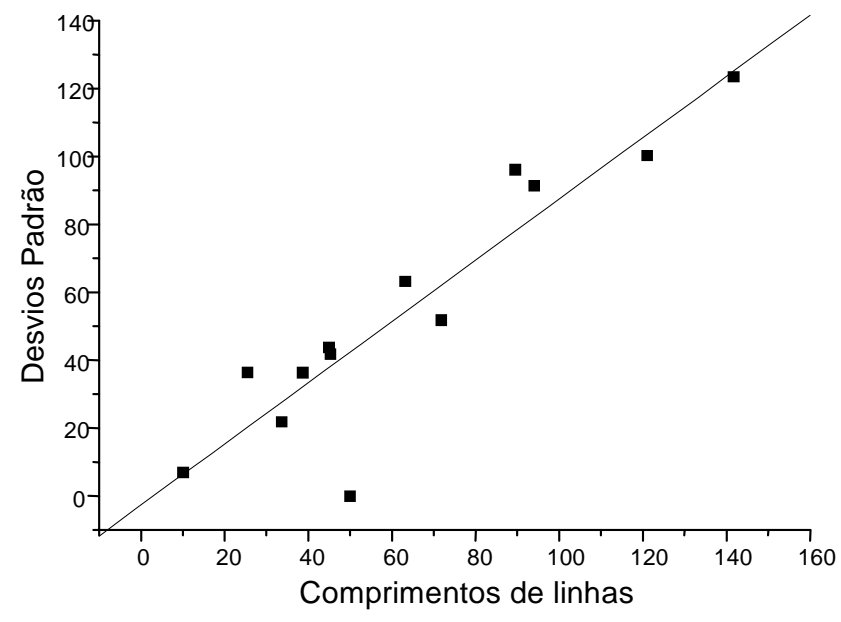

Figura 1 - Desvio padrão da média geométrica em função da média geométrica dos emparelhamentos de comprimentos de linha do estresse frente aos cuidados de enfermagem

Na Figura 1, observa-se que a relação é linear, de modo que quanto maior o emparelhamento de comprimento de linhas tanto maior é o desvio padrão, ou seja, a variabilidade das estimativas é uma função linear da magnitude do emparelhamento, mostrando, assim, o princípio geral conhecido como lei de Ekman.

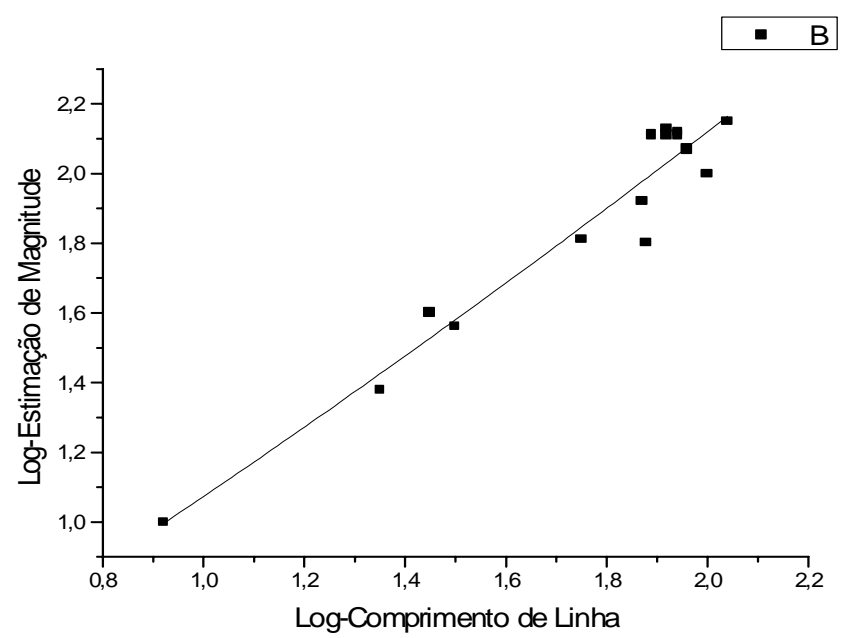

Figura 2 - Relação entre os logaritmos das médias geométricas das estimativas de magnitudes e as médias geométricas dos emparelhamentos de comprimentos de linhas da atribuição de estresse frente ao cuidado de enfermagem

$\mathrm{Na}$ Figura 2, as médias geométricas dos emparelhamentos de comprimentos de linhas são 
projetadas em coordenadas logarítmicas em função das correspondentes médias geométricas das estimativas de magnitudes para cada técnica de enfermagem. Ainclinação dessa linha reta é igual a 1,05. Esse valor é muito próximo do que aquele predito ${ }^{(10)}$, quando estão envolvidos diretamente emparelhamento de comprimento de linhas e de estimação de magnitude.

O expoente é o parâmetro mais importante, pois determina a curva que representa a relação entre estímulo e resposta. Se o expoente é exatamente igual a 1,0, a função segue uma linha reta. Nesse caso, a magnitude da sensação registrada varia linearmente com a intensidade do estímulo. Os psicofísicos tiveram como preocupações fundamentais a possibilidade de mensurar as sensações provocadas por estímulos físicos e a maneira de proceder à mensuração ${ }^{(10)}$.

\section{CONCLUSÕES}

Os dados obtidos neste estudo permitem concluir que:

1. os cuidados sondagem vesical de demora, sondagem nasogástrica, sondagem vesical de alívio, coleta de sangue e soroterapia foram considerados aqueles de maior atribuição de estresse, enquanto que os cuidados verificação de temperatura, colocação de luvas estéreis, arrumação de leito e oxigenoterapia os de menor atribuição em todas as escalas obtidas pelos diferentes métodos psicofísicos diretos;

2. as ordenações resultantes de todos os métodos produzem posições de atribuição ao estresse altamente

\section{REFERÊNCIAS BIBLIOGRÁFICAS}

1. Hanson GF. Refocusing the skills laboratory. Nurse Educ 1993; 18(2):10-2.

2. Selye H. The stress of life. New York (USA): Mc Graw-Hill; 1956.

3. Selye H. Stress: a tensão da vida. São Paulo (SP): Ibrasa; 1959.

4. Chaves EC. Stress e trabalho do enfermeiro: a influência de características individuais no ajustamento e tolerância ao turno noturno. [tese]. São Paulo (SP): Instituto de Psicologia/ USP; 1994.

5. Menzies IEP. Nurses under stress. Int Nurs Rev 1960; 7(6):916. concordantes para os diferentes cuidados de enfermagem; 3. a variabilidade das estimativas indicada pelo desvio padrão geométrico é uma função linear das médias das estimativas de magnitudes (ou das de emparelhamento de comprimentos de linhas), confirmando que a lei de Ekman é válida também para contínuos não-métricos;

4. a relação entre as estimativas de magnitudes e as estimativas do emparelhamento de comprimentos de linhas é uma função de potência com um expoente não significantemente diferente de 1,00;

5. a escala de atribuição de estresse frente aos cuidados de enfermagem é válida, estável e consistente, pois o expoente obtido através dos emparelhamentos de comprimentos de linhas e de estimativas de magnitudes não foi significantemente diferente do expoente predito pela propriedade de transitividade das escalas, ou seja, daquele predito por Stevens.

Em resumo, os resultados fornecem uma escala de atribuição de estresse frente aos cuidados de enfermagem, em nível de mensuração de razão, que é válida, estável e consistente. A escala de razão de atribuição de estresse frente aos cuidados de enfermagem, baseada nos julgamentos obtidos nos dois experimentos, para as duas diferentes amostras, mostra ordenações muito próximas umas das outras, sendo consideradas as de maior atribuição os cuidados sondagem vesical de demora, sondagem nasogástrica, sondagem vesical de alívio, coleta de sangue e soroterapia, enquanto que os cuidados verificação de temperatura, colocação de luvas estéreis, arrumação de leito e oxigenoterapia as de menor atribuição. A concordância entre valores escalares é elevada, indicando que as escalas são homogêneas e consistentes.

6. Filho PCPT, Pires E, Araújo GA. Características evidenciáveis de estresse em discentes de enfermagem. Rev Latino-am Enfermagem 1999 abril; 7(2):91-3.

7. Malasch C. Job burnout: how people cope. In: Mc Connell $E A$, organizador. Burnout in the nursing profession: coping, strategies, causes and cost. Saint Louis (USA): Mosby; 1982. 8. Bianchi ERF. Stress entre Enfermeiros Hospitalares. [tese]. São Paulo (SP): Escola de Enfermagem/USP; 1999.

9. Masuda M, Holmes TH. Magnitude estimation of social readjustments. Psychosom Res 1967; 11:219-25.

10. Faleiros Sousa FAE. Mensuração de atributos sociais e clínicos: um enfoque experimental. Ribeirão Preto (SP): FUNPEC; 2000.

Recebido em: 10.10 .2003

Aprovado em: 6.5.2004 